\title{
Glueball Matrix Elements on Anisotropic Lattices*
}

\author{
Y. Chen ${ }^{\text {ab }}$, S.-J. Dong ${ }^{\mathrm{a}}$, T. Drapera ${ }^{\mathrm{a}}$ I. Horváth ${ }^{\mathrm{a}}$, F.-X. Lee ${ }^{\mathrm{cd}}$, N. Mathur ${ }^{\mathrm{a}}$, C. Morningstare, \\ M. Peardon ${ }^{\text {f }}$ S. Tamhankar ${ }^{\mathrm{a}}$, B.L. Young ${ }^{\mathrm{g}}$, and J.-B. Zhang ${ }^{\mathrm{h}}$

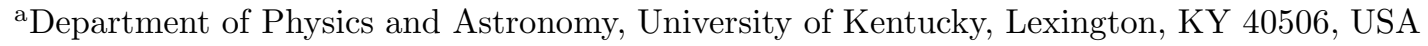 \\ ${ }^{\mathrm{b}}$ Institute of High Energy Physics, Chinese Academy of Sciences, Beijing 100039, P. R. China \\ ${ }^{\mathrm{c}}$ Center for Nuclear Studies, Dept. of Physics, George Washington Univ., Washington, DC 20052, USA \\ d Jefferson Lab, 12000 Jefferson Avenue, Newport News, VA 23606, USA \\ ${ }^{\text {e}}$ Department of Physics, Carnegie Mellon University, Pittsburgh, PA 15213, USA \\ ${ }^{\mathrm{f}}$ School of Mathematics, Trinity College, Dublin 2, Ireland \\ 'Department of Physics, Carnegie Mellon University, Pittsburgh, PA 15213, USA \\ ${ }^{\mathrm{h}}$ CSSM and Department of Physics, Univ. of Adelaide, Adelaide, SA 5005, Australia
}

\begin{abstract}
The glueball-to-vacuum matrix elements of local gluonic operators in scalar, tensor, and pseudoscalar channels are investigated numerically on several anisotropic lattices with the spatial lattice spacing in the range $0.1 \mathrm{fm}$ $-0.2 \mathrm{fm}$. These matrix elements are needed to predict the glueball branching ratios in $J / \psi$ radiative decays which will help to identify the glueball states in experiments. Two types of improved local gluonic operators are constructed for a self-consistent check, and the finite volume effects are also studied. The lattice spacing dependence of our results is very small and the continuum limits are reliably extrapolated.
\end{abstract}

\section{Introduction}

Glueballs, predicted by QCD, are so exotic from the point of view of the naive quark model that their existence will be a confirmation of QCD. Extensive numerical studies have been carried out to simulate the glueball spectrum and resulted that the low-lying glueballs are in the mass range $1-3 \mathrm{GeV}$, which suggests that the $J / \psi$ radiative decays are the ideal hunting ground for glueballs. There are several possible glueball candidates in the final states of $J / \psi$ radiative decays, however, more criteria are needed for their unambiguous identifications, one of which might be the partial widths of $J / \psi$ radiative decays into glueballs. To estimate these partial widths, the vacuum-to-glueball transition matrix elements(TME) of local gluonic operators should

*Presented by S.-J. Dong. be derived first.

The techniques of lattice simulations in the glueball sector have been substantially improved in the past decade. Inspired by the success of anisotropic lattice techniques in the simulations of the glueball spectrum [1] and as a continuation of former studies [23], this work is devoted to the numerical study of TME on anisotropic lattices with tadpole-improved gauge action.

\section{LOCAL GLUONIC OPERATORS}

The TME computed in this work are $\left\langle 0|S(x)| 0^{++}\right\rangle,\left\langle 0\left|T_{\mu \nu}(x)\right| 2^{++}\right\rangle$, and $\left\langle 0|P(x)| 0^{-+}\right\rangle$, where $\left|J^{P C}\right\rangle$ refers to the glueball state with the quantum number $J^{P C}$, and the local operators $S(x), T_{\mu \nu}$, and $P(x)$ are trace anomaly $g^{2} \operatorname{Tr} G_{\mu \nu} G_{\mu \nu}(x)$, the energy-momentum tensor $g^{2} \operatorname{Tr}\left(G_{\mu \alpha}(x) G_{\alpha \nu}(x)-\frac{1}{4} g_{\mu \nu} G^{2}(x)\right)$, and the topological charge density $g^{2} \epsilon_{\mu \nu \rho \sigma} \operatorname{Tr} G_{\mu \nu}(x) G_{\rho \sigma}(x)$, 
Table 1

The simulation parameters.

\begin{tabular}{ccccrr}
\hline$\beta$ & $\xi$ & $u_{s}$ & $a_{s}(\mathrm{fm})$ & $L^{3} \times T$ & \#Meas. \\
\hline 2.4 & 5 & 0.409 & $0.222(2)$ & $8^{3} \times 40$ & 20000 \\
& & & & $12^{3} \times 64$ & 10000 \\
& & & & $16^{3} \times 80$ & 10000 \\
2.6 & 5 & 0.438 & $0.176(1)$ & $12^{3} \times 64$ & 8600 \\
2.7 & 5 & 0.451 & $0.156(1)$ & $12^{3} \times 64$ & 10000 \\
3.0 & 3 & 0.500 & $0.120(1)$ & $16^{3} \times 48$ & 10000 \\
3.2 & 3 & 0.521 & $0.101(1)$ & $24^{3} \times 72$ & 7900 \\
\hline
\end{tabular}

respectively $\left(G_{\mu \nu}(x)\right.$ is the gauge field strength and $g$ the gauge coupling). Two types of lattice local gluonic operators (Type-I and Type-II) are constructed in this work.

Type-I operators are linear combinations of a set of small Wilson loops according to the irreducible representations of the lattice symmetry group, namely, $A_{1}^{++}, A_{1}^{-+}, E^{++}$, and $T_{2}^{++}$4. Letting $O^{R}(x)$ be the local operators of specific quantum number $R$, the construction of Type-I operators can be expressed as

$O^{R}(x)=\sum_{i} C_{i}^{R} \operatorname{Re} \operatorname{Tr}\left(W_{1}^{(i)}(x)+\alpha W_{2}^{(i)}(x)+\ldots\right),(1)$

where $C_{i}^{R}$ are the combinational coefficients. Different Wilson loops $W_{1}, W_{2}, \ldots$ are included with proper factors $\alpha$ to improve the operator.

To construct Type-II operators, we define the lattice gauge field strength $\hat{F}_{\mu \nu}(x)$ as

$\hat{F}_{\mu \nu}(x)=\operatorname{Im}\left\langle f\left(u_{s}\right) P_{\mu \nu}(x)+g\left(u_{s}\right) R_{\mu \nu}(x)\right\rangle_{c}$,

where $P_{\mu \nu}(x)$, and $R_{\mu \nu}(x)$ are respectively the plaquette and rectangle at $x . \quad\langle\mid\rangle_{c}$ means the clover average, $f\left(u_{s}\right)$ and $g\left(u_{s}\right)$ are factors including the tadpole parameter $u_{s}=\left(\left\langle 1 / 3 \operatorname{Tr} P_{i j}\right\rangle\right)^{1 / 4}$ and are chosen in a way that yields $\hat{F}_{\mu \nu}(x)=$ $a_{s}^{2}\left(G_{\mu \nu}(x)+O\left(a_{s}^{4}\right)\right)\left(a_{s}\right.$ is the spatial lattice spacing). Proper combinations of $\operatorname{Tr} \hat{F}_{\mu \nu}(x) \hat{F}_{\rho \sigma}(x)$ give the Type-II operators with reduced lattice artifacts.

There are seven matrix elements calculated in this work, which are denoted by $(\mathrm{S}, \mathrm{B}),(\mathrm{S}, \mathrm{E})$, $(\mathrm{E}, \mathrm{B}),(\mathrm{E}, \mathrm{E}),(\mathrm{T}, \mathrm{B}),(\mathrm{T}, \mathrm{E})$, and $(\mathrm{PS})$. Here $(, \mathrm{B})$ (or $(, \mathrm{E})$ ) means the operator is made up of colormagnetic (or color-electric) field, and (PS) refers to the pseudoscalar channel.

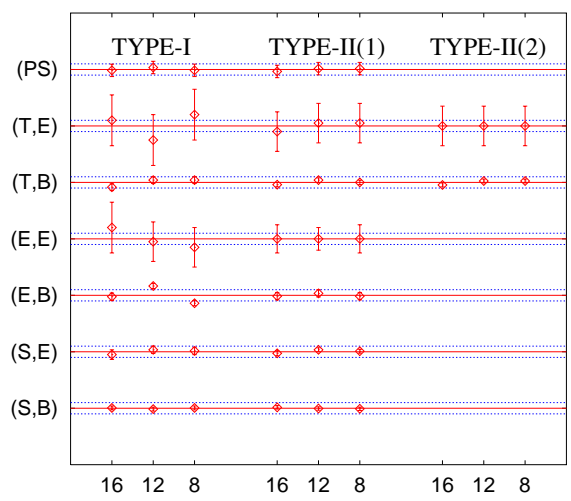

Figure 1. Finite-volume effects of matrix elements.

\section{SIMULATION DETAILS AND RE- SULTS}

Numerical simulations were carried out on anisotropic lattices with tadpole-improved gauge action 11. Five independent simulations have been done with input parameter listed in Table. 1.

With the tadpole improvement, the local gluonic operators on the lattice are all improved to $O\left(a_{s}^{4}\right)$ ( $a_{s}$ is the spatial lattice spacing) at the tree level. With applying the variational method to the combinations of smeared Wilson loops with different prototypes 1], glueball states are obtained through correlators of smeared operators which have large overlaps with glueball states. Six independent runs were carried out, on lattices with spatial lattice spacings in the range 0.1 $f m-0.22 \mathrm{fm}$, to measure the smeared-smeared correlators $C_{S S}(t)$ and smeared-local correlators $C_{S L}(t)$. The matrix elements are extracted by fitting $C_{S S}(t)$ and $C_{S L}(t)$ simultaneously using the correlated $\chi^{2}$ method. The fit models are taken as

$C_{S S}(t)=X^{2} e^{-M t}$
$C_{S L}(t)=X Y e^{-M t}$,

where $X$ is the amplitude of the glueball operators, $Y$ is the glueball-to-vacuum matrix element, 


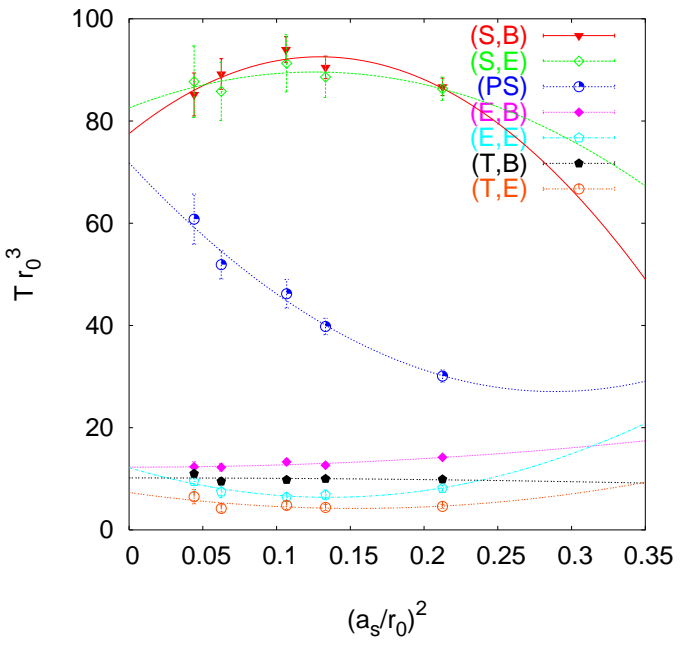

Figure 2. Continuum extrapolations of matrix elements of Type-I operators.

and $M$ is the glueball mass.

The finite volume effects (FVE) of matrix elements are studied on lattices $8^{3} \times 40,12^{3} \times 64$, and $16^{3} \times 80$ at $\beta=2.4, \xi=5$, and the results are shown in Fig. 1] where each point with a errorbar is the fractional change, $\delta_{G}(L)=1-f_{G}(L) / \bar{f}_{G}$, in the matrix elements $\left(\bar{f}_{G}\right.$ is the average value of the matrix elements of glueball $G$ over the three lattice volumes, and $f_{G}(L)$ is the matrix element of glueball $G$ measured on lattice $L^{3} \times T$ ). The labels $L=8,12$, and 16 denote the different lattice volumes, and the labels along the vertical axis represent the matrix elements of the different local operators. To guide eyes, $\delta_{G}=0$ and $\delta_{G}= \pm 0.02$ are also drawn in Fig. 11 with solid line and dash lines, respectively. All changes are statistically consistent with zero, indicating that systematic errors from FVE are negligible.

The simulated results of matrix elements at different lattice spacings are shown in Fig. 2(Type-I operators) and Fig. 3 (Type-II operators), where $a_{s}$ (in units of $r_{0} \sim(410(20) M e V)^{-1}$, the hadronic scale parameter) dependences are observed. The matrix elements can be reliably extrapolated to the continuum limit by using the form $f\left(a_{s} / r_{0}\right)=T r_{0}^{3}+c_{2}\left(a_{s} / r_{0}\right)^{2}+c_{4}\left(a_{s} / r_{0}\right)^{4}$, where $T r_{0}^{3}, c_{2}$, and $c_{4}$ are best-fit parameters. We keep the $a_{s}^{2}$ term in the fitting model because there are residual $\alpha_{s} a_{s}^{2}$ artifacts in the gauge ac-

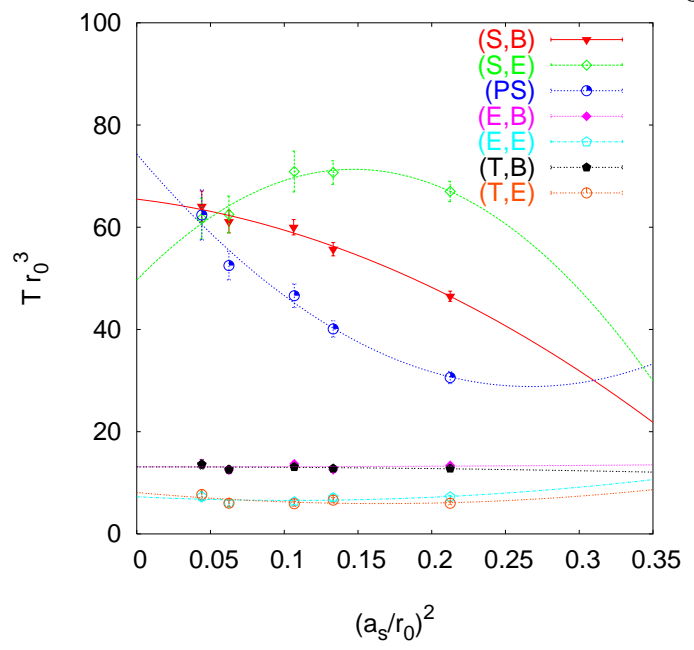

Figure 3. Continuum extrapolations of matrix elements of Type-II operators.

tion and some local operators. From the figures one can find that the Type-II operators exhibit better behaviors (for example, the matrix elements of $T_{2}$ and $E$ representations coincide, as it should be when the rotational symmetry is restored in the continuum limit).

The physically available predictions will not be derived until the local gluonic operators are properly renormalized. The nonperturbative renormalization of these operators is in progress.

This work is supported by DOE Grants DEFG05-84ER40154 and DE-FG02-02ER45967. Y. Chen is partly supported by NSFC (No.10075051, 12035040) and CAS (KJCX2-SW-N02).

\section{REFERENCES}

1. C. Morningstar and M. Peardon, Phys. Rev. D56 (1997) 4043; Phys. Rev. D60 (1999) 034509 .

2. Y. Liang, K.F. Liu, B.A. Li, and S.J. Dong, Phys. Lett. B307 (1993) 375.

3. S.J. Dong et al, Nucl. Phys. Proc. Suppl. 63 (1998) 254.

4. B. Berg and A. Billoire, Nucl. Phys. B221 (1983) 109. 